\title{
Genetic Factors and Orofacial Motor Learning Selectively Influence Variability in Central Sulcus Morphology in Chimpanzees (Pan troglodytes)
}

\author{
William D. Hopkins, ${ }^{1,2,3,4}$ @Oliver Coulon, ${ }^{5} \oplus^{-}$Adrien Meguerditchian, ${ }^{4,6}$ Michelle Autrey, ${ }^{2}$ Kendall Davidek, ${ }^{2}$ \\ Lindsay Mahovetz, ${ }^{2}$ Sarah Pope, ${ }^{2}$ Mary Catherine Mareno, ${ }^{7}$ and Steven J. Schapiro ${ }^{7}$ \\ ${ }^{1}$ Division of Developmental and Cognitive Neuroscience, Yerkes National Primate Research Center, Atlanta, Georgia 30322, ${ }^{2}$ Neuroscience Institute and \\ Language Research Center, Georgia State University, Atlanta, Georgia 30302, ${ }^{3}$ IMéRA Institut d'Etudes Avancées, Aix-Marseille University, 13004 Marseille, \\ France, ${ }^{4}$ Brain Language Research Institute, Aix-Marseille University, Centre National de la Recherche Scientifique (CNRS), 13604 Aix-en-Provence, \\ France, ${ }^{5}$ Laboratoire des Sciences de l'Information et des Systèmes, Unité Mixte de Recherche (UMR) 7296, Aix-Marseille Universite, Centre Nationale de la \\ Recherche Scientifque, Institut de Neuroscience de La Timone, Marseille, France 13288, ${ }^{6}$ Laboratoire de Psychologie Cognitive, UMR 7290, Aix-Marseille \\ University, CNRS, Marseille, France, and ${ }^{7}$ National Center for Chimpanzee Care, Department of Veterinary Sciences, The University of Texas M.D.
}

Anderson Cancer Center, Bastrop, Texas 78602

Captive chimpanzees (Pan troglodytes) have been shown to learn the use of novel attention-getting (AG) sounds to capture the attention of humans as a means of requesting or drawing their attention to a desired object or food. There are significant individual differences in the use of AG sounds by chimpanzees and, here, we examined whether changes in cortical organization of the central sulcus (CS) were associated with AG sound production. MRI scans were collected from 240 chimpanzees, including 122 that reliably produced AG sounds and 118 that did not. For each subject, the depth of CS was quantified along the superior-inferior plane with specific interest in the inferior portion corresponding to the region of the motor cortex where the mouth and orofacial movements are controlled. Results indicated that CS depth in the inferior, but not superior, portion was significantly greater in chimpanzees that reliably produced AG sounds compared with those who did not. Quantitative genetic analyses indicated that overall CS surface area and depth were significantly heritable, particularly in the superior regions, but less so in the inferior and central portions. Further, heritability in CS depth was altered as a function of acquisition of AG sounds. The collective results suggest that learning to produce AG sounds resulted in region-specific cortical reorganization within the inferior portion of the $\mathrm{CS}$, a finding previously undocumented in chimpanzees or any nonhuman primate.

Key words: central sulcus; chimpanzee; heritability; language; orofacial movements; vocal learning

Significance Statement

Recent studies in chimpanzees (Pan troglodytes) have shown that some can learn to produce novel sounds by configuring different orofacial movement patterns and these sounds are used in communicatively relevant contexts. Here, we examined the neuromorphological correlates in the production of these sounds in chimpanzees. We show that chimpanzees that have learned to produce these sounds show significant differences in central sulcus (CS) morphology, particularly in the inferior region. We further show that overall CS morphology and regions within the superior portion are significantly heritable, whereas central and inferior portions of the CS are not. The collective findings suggest chimpanzees exhibit cortical plasticity in regions of the brain that were central to the emergence of speech functions in humans.

\section{Introduction}

In primate brains, the central suclus (CS) separates primary motor and somatosensory cortex. Electrical stimulation of the precentral gyrus elicits different motor movements that are topographically organized along the superior-inferior plane in both human and nonhuman primate brains (Penfield and Boldrey, 
1936; Bailey et al., 1950). For example, as in humans, Bailey et al. (1950) reported that the chimpanzee (Pan troglodytes) CS has superior and inferior "knees" that serve as boundaries between the leg and arm movements and arm and face movements (Fig. 1). Stimulation of the ventral region of the CS immediately below the inferior knee elicited movements of the lips, mouth, and larynx, whereas stimulation of the cortex above the superior knee elicited movements of the legs and trunk.

Here, we examined the role of genetic and nongenetic factors on variation in CS morphology in chimpanzees. The CS is a highly conserved sulcus in primate evolution, so its development is likely under some genetic control (Hopkins et al., 2014b; Gómez-Robles et al., 2015). Indeed, McKay et al. (2013) found that heritability in CS depth is highest in the superior and inferior regions and essentially absent in the central portion. Similarly, GómezRobles et al. (2015) reported that the spatial location of the superior and inferior points of the CS are significantly heritable in both humans and chimpanzees. Similar to McKay et al. (2013), we used quantitative genetics to evaluate heritability in the overall surface area and mean depth along 100 equally space regions along the superior-inferior plane in a sample of captive chimpanzees.

The second purpose of this study was to determine whether individual differences in the use of attention-getting (AG) sounds were associated with variation in the depth of the CS in chimpanzees, particularly within the inferior portion of the sulcus. Studies have demonstrated that some chimpanzees and other great apes voluntarily produce several classes of novel sounds to capture the attention of an otherwise inattentive audience (Theall and Povinelli, 1999; Liebal et al., 2004; Poss et al., 2006; Cartmill and Byrne, 2007; Leavens et al., 2008; Gentry et al., 2015). Experimental evidence has shown that the use of AG sounds is under voluntary control (Hopkins et al., 2007; Hopkins et al., 2011), often occurs in conjunction with manual gestures (Hopkins and Cantero, 2003) and grooming (Leavens et al., 2014; Fedurek et al., 2015; Watts, 2016), is lateralized to the left hemisphere (Losin et al., 2008; Wallez et al., 2012), and is socially learned (Taglialatela et al., 2012). For instance, systematic investigations of the production of AG sounds in 279 chimpanzees from two different populations found that $52 \%$ and $46 \%$ of the chimpanzees within each sample reliably produce some type of AG sound, respectively (Hopkins et al., 2010; Taglialatela et al., 2012). Therefore, some chimpanzees reliably produce AG sounds and others do not.

The neural and potential genetic mechanisms that underlie individual variation in AG sound production remains unstudied and here we explored this question in two ways. First, we investigated whether chimpanzees that do or do not reliably produce

American Psychological Association guidelines for the ethical treatment of animals were adhered to during all aspects of this study. We thank the veterinary staff at the NCCC and YNPRC for assistance in the collection of the MRI scans.

The authors declare no competing financial interests.

Correspondence should be addressed to William D. Hopkins, Neuroscience Institute, Georgia State University, P.0. Box 5030, Atlanta, GA 30302. E-mail: whopkin@emory.edu or whopkins4@gsu.edu.

DOI:10.1523/JNEUROSCI.2641-16.2017

Copyright $\odot 2017$ the authors $\quad 0270-6474 / 17 / 375476-09 \$ 15.00 / 0$
AG sounds differed in overall CS depth or asymmetry. In light of the fact the AG sounds are socially learned, we were specifically interested in assessing whether differences in overall CS depth or asymmetry were specific to the inferior regions, where the mouth, lips, and larynx are located in the motor cortex (Bailey et al., 1950; Fig. 1). Second, we used quantitative genetic analyses to test for genetic correlations between AG sound production in CS depth along and superior-inferior plane. If common genes underlie both the production of AG sounds and CS depth, particularly in the inferior regions, then this would suggest that genetic mechanisms underlie their expression. Alternatively, if phenotypic associations are evident between AG sound production and region-specific CS depth and these are not attributable to common genetic factors, then this may indicate changes in CS morphology that are experience dependent.

\section{Materials and Methods}

Subjects. For the quantitative genetic analyses, MRIs were obtained from 264 captive chimpanzees housed at the Yerkes National Primate Research Center (YNPRC) and the National Center for Chimp Care (NCCC) of The University of Texas M.D. Anderson Cancer Center. There were 152 females and 112 males ranging from 6 to 53 years of age. Within this sample, there were 132 mother-reared, 79 human-reared, and 53 wildborn individuals. Within the entire sample, behavioral data were available for 251 individuals, including 148 females and 103 males. Therefore, the analyses focusing on the association between CS depth, AG sound production, and gesture handedness were performed on a smaller sample than the quantitative genetic analyses. The methods for measuring hand preferences for manual gestures and the production of AG sounds by the chimpanzees have been described in detail previously (Hopkins et al., 2005; Taglialatela et al., 2012). For gesture handedness, there were 81 left-handed, 51 ambiguously handed, and 119 right-handed chimpanzees. In terms of AG sound production, 123 individuals were classified as never producing $\mathrm{AG}$ sounds $\left(\mathrm{AG}^{-}\right)$and the remaining 128 chimpanzees were observed to produce at least one AG sound within the testing paradigm $\left(\mathrm{AG}^{+}\right)$. Within the $\mathrm{AG}^{+}$group, the number of sounds produced in 6 test trials ranged between 1 and $6($ mean $=3.72, \mathrm{SE}=0.031)$.

MRI image collection. Both in vivo and postmortem MRI scan data were used in this study. In vivo scans were obtained at the time the 


\section{Pipeline}

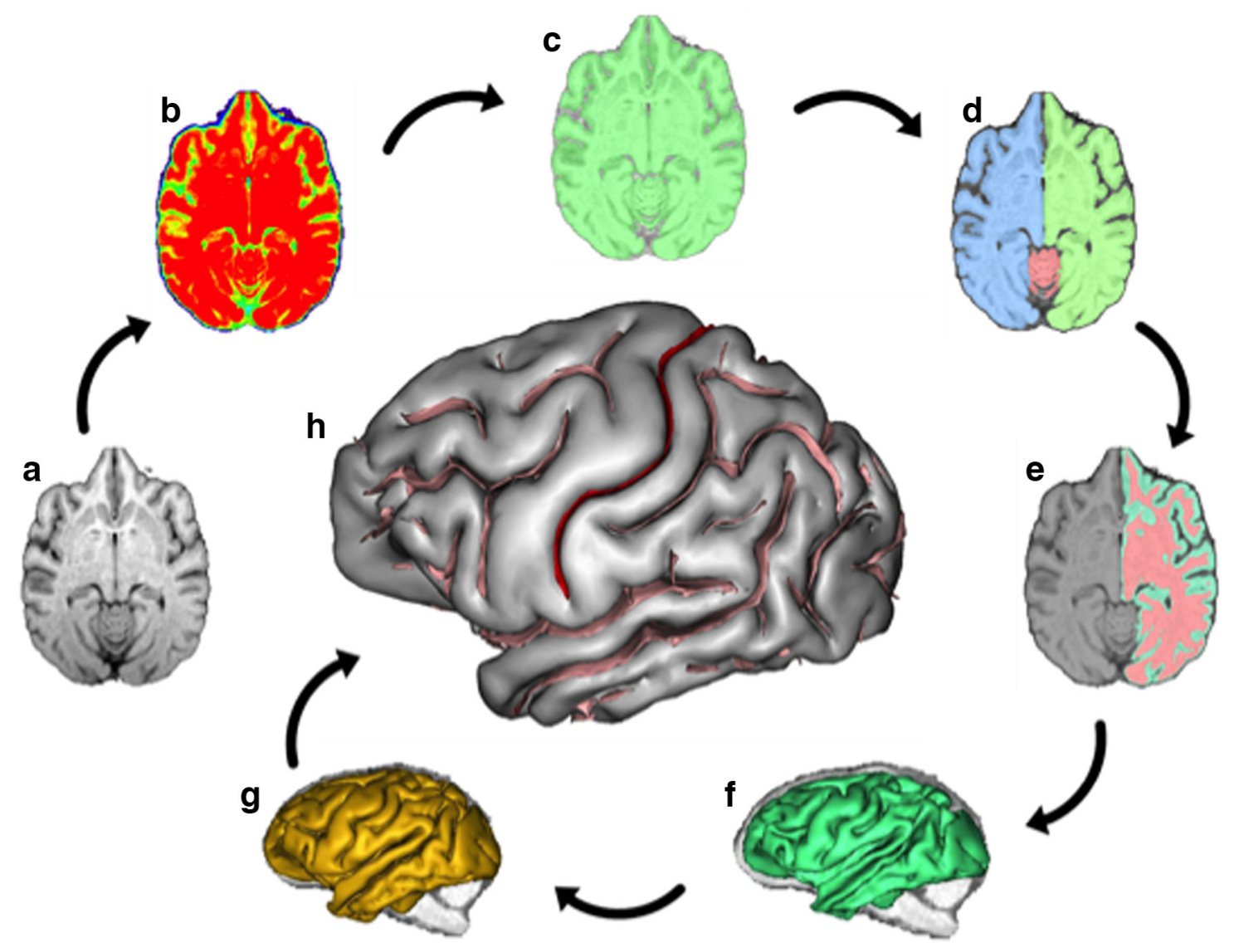

Figure 2. The sequence of postimage processing steps performed from the raw T1-weighted MRI scans (see text for description). The CS (red) is labeled on the 3D cortical graph output.

chimpanzees were being surveyed for their annual physical examinations. Subjects were first immobilized by ketamine $(10 \mathrm{mg} / \mathrm{kg})$ or telazol (3-5 mg/kg) and subsequently anesthetized with propofol (40-60 mg/ $\mathrm{kg} / \mathrm{h}$ ) following standard procedures at the YNPRC and NCCC facilities. YNPRC subjects were then transported to the MRI facility and NCCC subjects were wheeled to the mobile imaging unit. The subjects remained anesthetized for the duration of the scans, as well as during the time needed to transport them between their home cage and the imaging facility (between 5 and10 $\mathrm{min}$ ) or mobile imaging unit (total time $\sim 5$ $\min$ ). Subjects were placed in the scanner chamber in a supine position with their head fitted inside the human-head coil. Scan duration ranged between 40 and $60 \mathrm{~min}$ as a function of brain size.

Seventy-eight chimpanzees were scanned using a 3.0 T scanner (Trio; Siemens) at YNPRC. T1-weighted images were collected using a 3D gradient echo sequence (pulse repetition $=2300 \mathrm{~ms}$, echo time $=4.4 \mathrm{~ms}$, number of signals averaged $=3$, matrix size $=320 \times 320$, voxel dimension $0.6 \mathrm{~mm} \times 0.6 \mathrm{~mm} \times 0.6 \mathrm{~mm}$ ). In addition, 142 NCCC chimpanzees were scanned using a $1.5 \mathrm{~T}$ Philips machine. T1-weighted images were collected in the transverse plane using a gradient echo protocol (pulse repetition $=19.0 \mathrm{~ms}$, echo time $=8.5 \mathrm{~ms}$, number of signals averaged $=$ 8 , and a $256 \times 256$ matrix, voxel dimension $.7 \mathrm{~mm} \times 0.7 \mathrm{~mm} \times 1.2 \mathrm{~mm}$ ). After completing MRI procedures, the subjects were temporarily housed in a single enclosure for 6-12 h to allow the effects of the anesthesia to wear off, after which time they were returned to their social group. Postmortem T2-scans were obtained from 44 chimpanzees that had died from natural causes. For the postmortem scanning, either 4.7 or $7 \mathrm{~T}$ magnets were used and T2-weighted images were collected in the transverse plane using a gradient echo protocol (voxel dimension $0.67 \mathrm{~mm} \times$
$0.67 \mathrm{~mm} \times 0.67 \mathrm{~mm}$ ) pulse repetition $=22.0 \mathrm{~s}$, echo time $=78.0 \mathrm{~ms}$, number of signals averaged $=8-12$, and a $256 \times 192$ matrix reconstructed to $256 \times 256$ ).

Sulci extraction and measurement. The sequence of postimage processing steps performed on the images are shown in Figure 2, $a-h$. The pipeline of processing used to extract the sulci from the raw T1-weighted image derives from a pipeline initially dedicated to the human brain and freely distributed as a BrainVISA toolbox (http://brainvisa.info) (Mangin et al., 2004). To account for the differences in chimpanzee anatomy compared with humans, a number of adjustments were performed before the scans were processed using the pipeline procedure within BrainVISA. Specifically, chimpanzee MRI volumes were skull stripped, cropped, and reformatted at $0.7 \mathrm{~mm}$ isotropic resolution using ANALYZE 11.0 software and subsequently imported into BrainVISA. The pipeline process of extracting the sulci from the cortex involved a number of steps (Mangin et al., 2004; Fig. $2 a-h$ ). To align the template brain, the anterior and posterior commissures were manually specified on the MRI at the point where they intersect with the midsagittal slice. The first step was to correct for spatial inhomogeneities in the signal intensity providing a spatially smooth bias field with a stable distribution of tissue intensities (Fig. $2 b$ ). Next, the analysis of the signal histogram and mathematical morphology were performed using an automatic analysis of the voxel intensities for the entire brain to obtain a binary mask of the brain (Fig. 2c). Adjustments were sometimes needed in the histogram process to determine gray and white matter means for chimpanzee brain scans. The mask was then split into the left and right hemispheres and the cerebellum (Fig. $2 d$ ). A negative mold of the white matter was computed from the split-brain mask. The outside boundary of this mold results 
from a $5 \mathrm{~mm}$ morphological closing of the masked hemisphere, filling up the folds. The gray/white interface is the inside boundary that preserves deformations and ensures the spherical topology of the mold (Fig. 2e). Finally the mold was skeletonized to detect cortical folding and topological constraints guaranteed that the resulting surfaces would have no holes (Mangin, 2000; Mangin et al., 2004; Fig. 2f,g). Finally, the folds making up the CS in each hemisphere were selected manually (Fig. $1 h$ ) using a 3D visualization interface by following standard anatomical landmarks (Bailey et al., 1950; Hopkins et al., 2014b) and the total surface area and average depth were computed for this sulcus in each hemisphere (Fig. 3).

Quantifying CS depth along the superior to inferior plane. The selected CS was meshed using a triangular mesh and the resulting surface was parameterized to create a longitudinal coordinate system (Coulon et al., 2006; Fig. $4 a-d)$. The parameterization process was constrained by the two ventral and dorsal sulcus extremities, automatically detected using the extrema of the first nonzero eigenfunction of the mesh Laplacian $\left(\mathrm{e}_{1}\right.$ and $\mathrm{e}_{2} ;$ Fig. $\left.4 b\right)$. From these points, a smooth and quasi-isometric (i.e., with minimal metric distortions) coordinate field was extrapolated, which localizes all mesh surface points according to their relative position along the sulcus between the two extremities (Fig. $4 c$ ). The coordinate field extends along the length of the CS from the superior/dorsal $(y=1)$ to the inferior/ventral $(y=100)$ ends of the sulcus. Depth was measured at 100 sulcal length positions in a superior-to-inferior progression along the parameterized sulcal mesh surface. Position 1 was adjacent to the interhemispheric fissure and position 100 was adjacent to the Sylvian fissure (Fig. 4c). At each position $y$ along the length, the depth was computed by measuring a geodesic distance (in millimeters) from the brain envelope to the fundus of the sulcus (Fig. 4d).

Data analysis. For each subject and hemisphere, we computed a total surface area and average depth of the CS (Fig. 3). In addition, we computed individual average surface area and average depth by summing the total left and right hemisphere values and dividing by two. For the parameterization analyses, rather than compare and estimate the total additive genetic variance $\left(h^{2}\right)$ scores for each of the 100 points along the sulcus within each hemisphere, we computed average depth values in intervals of five positions. Therefore, we computed 20 depth measures for each hemisphere along the superior-to-inferior plane (1-20). From these measures, we computed the mean depths between the hemispheres at each position as follows: mean depth $=(R+L) / 2$, as well as the difference scores in depth at each position as follows: difference $=(R-L)$. For the difference scores, positive values indicate rightward asymmetries and negative values indicated leftward biases. For all analyses, $\alpha$ was set to $p<0.05$ and post hoc analyses, when necessary, were performed using Tukey's honestly significant difference test.

Quantitative genetic analyses. To estimate heritability, we used the software package SOLAR (Almasy and Blangero, 1998). which uses a variance components approach to estimate the polygenic component of variance when considering the entire pedigree (Rogers et al., 2007; Fears et al., 2009; Fears et al., 2011; Hopkins et al., 2014a). $h^{2}$ is the proportion of total phenotypic variance that is attributable to all genetic sources. Total phenotypic variance is constrained to a value of 1 ; therefore, all nongenetic contributions to the phenotype are equal to $1-h^{2}$. We used SOLAR in two ways. First, we used SOLAR to determine heritability in the total surface area, average depth, and regional variation in CS depth for the left and right hemisphere. Second, we used the bivariate function within SOLAR to calculate genetic correlations between CS depth measures in the left and right hemispheres along the superior-inferior plane and between depth measures for the left and right hemisphere CS mea-

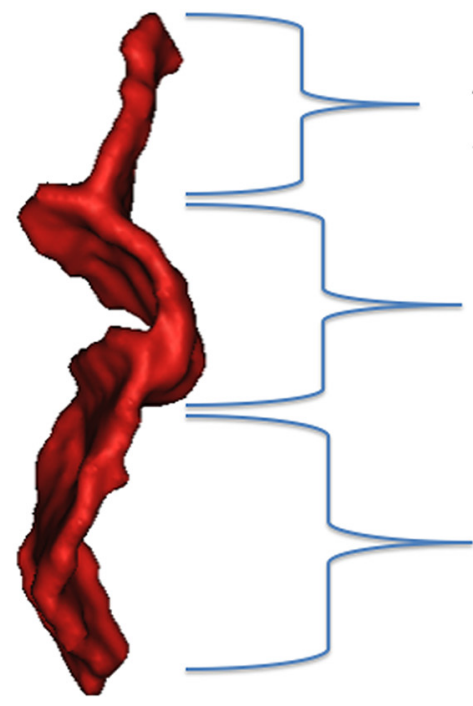

b
Leg

Trunk Shoulder

Arm

Hand

Thumb

Face Tongue Pharnyx Larynx

\section{Results \\ Descriptive data}

Because there are very little data available on CS morphology in nonhuman primates, we initially performed some descriptive analyses. Shown in Table 1 is the average overall CS surface area and mean depth for male and female chimpanzees scanned at 1.5, 3 , and 7 T. For the overall surface area and mean CS depth, we performed two mixed-model ANCOVAs with sex and rearing history serving as the between-group factors and scanner and age as the covariates. Hemisphere was the repeated measure. For surface area, we found a main effect for sex of $F_{(1,255)}=8.698, p=$ 0.003 . Not surprisingly, males had significantly larger CS surface areas than females. No other main effects or interactions were significant. However, we did find that the covariate scanner was significant $\left(F_{(1,259)}=17.145, p=0.000\right)$. The average surface area for chimpanzees scanned at $1.5 \mathrm{~T}$ was smaller than those scanned at 3 and $7 \mathrm{~T}$ (Table 1). For the mean CS depth, no significant main effects or interactions were found; however, as was the case with surface area, scanner was a significant covariate $\left(F_{(1,259)}=40.946\right.$, $p=0.000)$. The average mean CS depth was lower in chimpanzees scanned at $1.5 \mathrm{~T}$ compared with those scanned at 3 and $7 \mathrm{~T}$ (Table 1 ).

For the parameterization data, we performed two mixedmodel ANCOVAs with sex serving as the between-group factor and scanner and age as the covariates. For one analysis, the mean CS depth for the 20 regions was the repeated measure and, for the second analysis, the repeated measure was the CS difference score. For the mean CS depth, significant main effects for CS region $\left(F_{(19,4788)}=214.25, p=0.000\right)$ and $\operatorname{sex}\left(F_{(1,252)}=5.729\right.$, $p=0.017)$ were found, as well as a two-way interaction between sex and CS region $\left(F_{(19,4788)}=2.241, p=0.002\right)$. The mean CS depths for male and female chimpanzees are shown in Figure 5. First, for both males and females and consistent with previous reports, chimpanzees showed the classically described pattern of CS depth with higher peaks at the inferior and posterior regions 

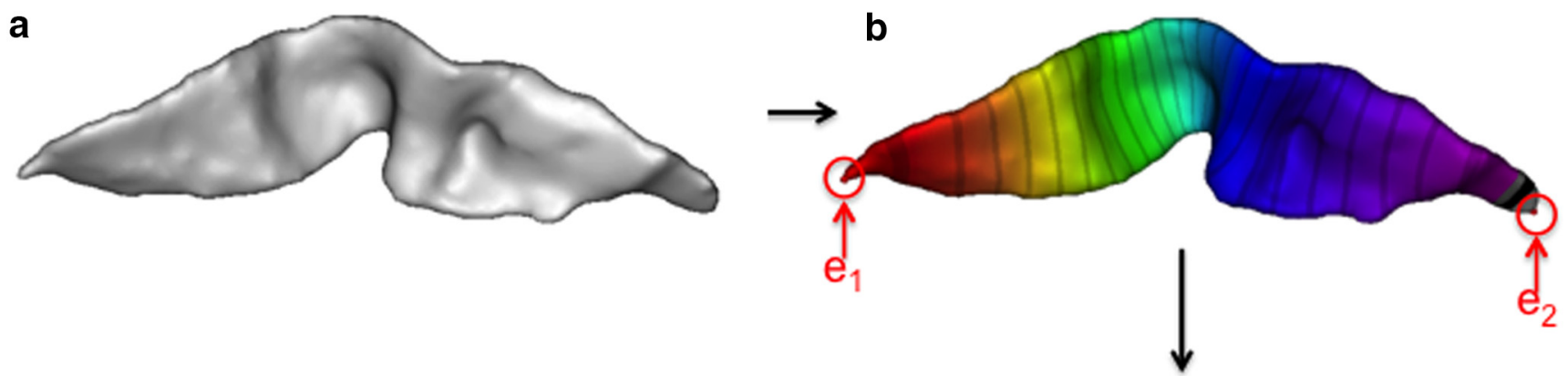

d


Figure 4. 3D rendering of the CS. $a, 3 \mathrm{D}$ rendering of the $(S$ extracted from the cortical graph and oriented on a horizontal axis where the left end of the sulcus represents the superior end $(y=0)$ and the right the inferior tip $(y=100)$. $\boldsymbol{b}$, Sulcus parameterized to create a normalized $x$-/ $y$-coordinate system with the bottom ridge of the sulcal mesh (i.e., the sulcal fundus; $x=100)$ and the top ridge (i.e., at the brain envelope; $x=1$ ) representing the $x$-axis. The end points of the sulcus where these top and bottom ridges join represent the $y$-axis. $c$, Depth measured from the brain envelope to the fundus of the sulcus along 100 sulcal length positions in a superior-to-inferior mesh surface. $\boldsymbol{d}$, Finally, from the output text file, we can determine the maximum superior point (SP) of the CS by finding the largest depth value between positions 1-50. The maximum inferior point (IP) is represented by the largest depth value found between positions 51 to 100 . The pli de passage frontoparietal moyen (PPFM) is the lowest depth value between the SP and IP positions.

Table 1. Descriptive data on CS surface area and mean depth

\begin{tabular}{llll}
\hline Scanner & \multicolumn{1}{l}{ Females } & Males & \multicolumn{1}{l}{ Mean } \\
\hline Surface area, $\mathrm{mm}^{2}$ & & & \\
1.5 T & $1410.39(20.80)$ & $1533.05(32.49)$ & $1417.72(20.80)$ \\
3 T & $1595.53(32.43)$ & $1643.26(72.77)$ & $1619.40(39.87)$ \\
7 T & $1580.10(53.02)$ & $1527.01(48.02)$ & $1553.56(35.71)$ \\
Mean & $1528.67(23.7)$ & $1567.77(31.02)$ & \\
Mean CS depth, mm & & & \\
1.5 T & $8.941(0.100)$ & $9.191(0.130)$ & $9.066(0.083)$ \\
3 T & $9.466(0.129)$ & $9.631(0.290)$ & $9.548(0.159)$ \\
7 T & $9.866(0.211)$ & $9.638(0.191)$ & $9.752(0.142)$ \\
Mean & $9.424(0.093)$ & $9.487(0.124)$ & \\
\hline
\end{tabular}

and shallower depth in the central portion. Second, with respect to the sex-by-region interaction, post hoc analysis indicated that males had larger CS depths at positions 4, 5, 6, 7, 8, and 20. We also found that the covariate scanner was significant $\left(F_{(2,252)}=\right.$ $7.553, p=0.001)$. The overall CS depth was lower in chimpanzees scanned at $1.5 \mathrm{~T}$ (mean $=12.88, \mathrm{SE}=0.074)$ compared with those scanned at $3 \mathrm{~T}($ mean $=13.36, \mathrm{SE}=0.104)$ and $7 \mathrm{~T}$ $($ mean $=13.17, \mathrm{SE}=0.135)$. For the CS difference scores, no significant main effects or interactions were found.

\section{Heritability in total CS surface area and depth}

In the initial analysis, heritability estimates in overall surface area and depth measures for the left and right hemisphere and the average between the two measures were derived using SOLAR. Covariates included sex, age, rearing history, and scanner. The results are shown in Table 2. Significant heritability was found for the overall, left, and right surfaces areas and average depths. Sex and scanner were significant covariates for the surface area and depth measures. As noted above, males had larger surface areas and depths than females and the chimpanzees scanned at $1.5 \mathrm{~T}$ had smaller surface areas and depths than chimpanzees scanned at 3.0 T and scanned postmortem. We also performed genetic

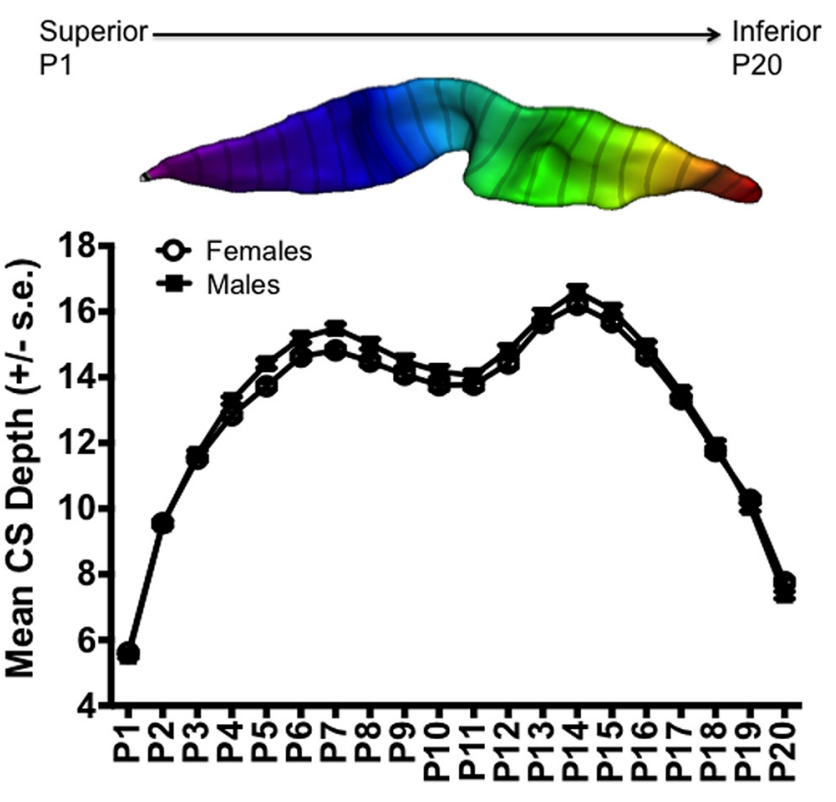

Figure 5. Mean depth (in millimeters) averaged from both the left and right hemispheres distributed along the superior-inferior $y$-axis of the CS for male chimpanzees (solid square) and female chimpanzees (open circle line).

correlations between the overall left and right surface areas and depth measures. For surface area, significant genetic (RhoG = $0.96, p=0.021)$ and environmental $(\mathrm{RhoE}=0.479, p=0.0002)$ correlations were found. Similarly, for mean depth, significant genetic $(\mathrm{RhoG}=0.98, p=0.021)$ and environmental $(\mathrm{RhoE}=$ $0.421, p=0.002)$ correlations were found.

\section{Heritability in CS depth from the superior to}

inferior positions

In the next set of analyses, we computed the heritability estimates for the parameterized depth measures that were computed along 
Table 2. Heritability in overall CS surface area and depth with covariates of scanner and sex $(N=264)$

\begin{tabular}{lllll}
\hline & $h^{2}$ & SE & $p$ & Variance \\
\hline $\begin{array}{l}\text { Surface area } \\
\text { Left }\end{array}$ & 0.374 & 0.119 & 0.0001 & \\
Right & 0.339 & 0.117 & 0.0002 & 0.099 \\
$\quad$ Mean & 0.403 & 0.118 & 0.0001 & 0.088 \\
$\begin{array}{l}\text { Mean depth } \\
\text { Left }\end{array}$ & 0.286 & 0.148 & 0.010 & \\
Right & 0.183 & 0.116 & 0.035 & 0.157 \\
Mean & 0.298 & 0.140 & 0.006 & 0.137 \\
\hline
\end{tabular}

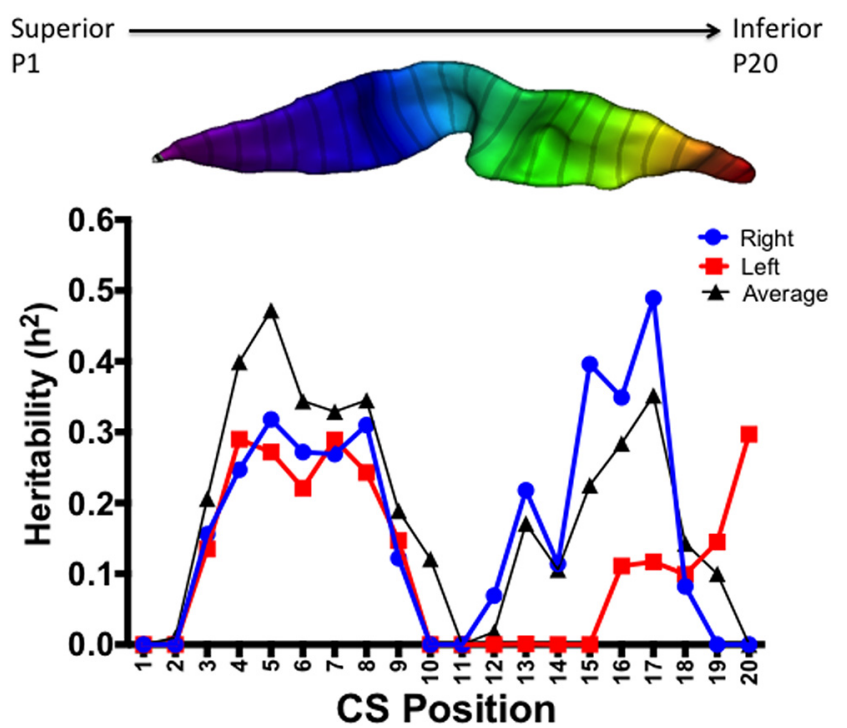

Figure 6. $h^{2}$ estimates for positions $1-20$ along the superior to inferior plane. Black triangle is the average for the left and right hemisphere, red square is the left hemisphere, and blue circle is the right hemisphere.

the superior-inferior plane. As before, age, sex, scanner, and rearing were covariates in each analysis. The $h^{2}$ estimates for each region of the 20 regions for the left and right hemispheres, as well as the average between the two, are shown in Figure 6. For the mean CS depth at each position, significant heritability was found at positions 3 to $8,15,16$, and 17 (Fig. 6). For the left hemisphere, significant heritability was found at positions 4 to 8 and 20. For the right hemisphere, significant heritability was found at positions 3 to 8 as well as positions 15, 16, and 17. Last, for the difference scores at each CS position, none of the heritability estimates was significant. Therefore, there was no evidence that asymmetries in CS depth were heritable.

Brain-behavior phenotypic associations

In the next analysis, we compared the average CS depth, $(R+$ $L) / 2$, and asymmetry in CS depth, R - L, at positions 1-20 in relation to sex, gesture handedness, and vocal grouping in two ANCOVAs. For each analysis, CS position was the repeated measure and sex, gesture handedness, and vocal grouping were the between-group factors. Age and scanner were covariates. For the average depths, we found a significant sex-by-region interaction $\left(F_{(17,3485)}=2.176, p=0.003\right)$. Post hoc analysis indicated that males had larger mean depth values than females for positions 3-7 and no significant differences were found for the remaining regions.

For the asymmetry measures, we found a significant main effect for gesture handedness $\left(F_{(2,237)}=4.117, p=0.016\right)$ and a

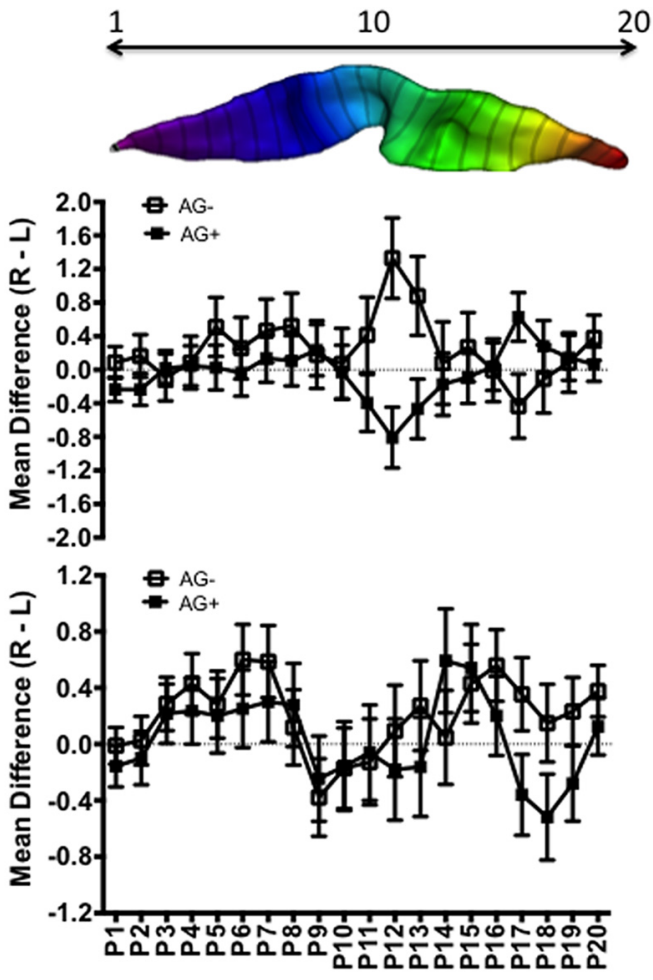

Figure 7. Mean ( $\pm \mathrm{SE}) \mathrm{CS}$ depth asymmetry values for positions $1-20$ for $\mathrm{AG}^{+}$and $\mathrm{AG}^{-}$ chimpanzees. $\boldsymbol{a}$, Males. $\boldsymbol{b}$, Females. On the top is a horizontal view of the CS from the superior $(0)$ to inferior (20) position to illustrate the location of the effect of AG sound use on variation in CS depth asymmetry.

significant three-way interaction between sex, vocal grouping, and region $\left(F_{(19,4503)}=1.826, p=0.015\right)$. For the gesture handedness main effect, post hoc analysis revealed that the difference scores were significantly lower in right-handed chimpanzees $($ mean $=-0.122)$ compared with ambiguously handed $($ mean $=$ $+0.304)$ and left-handed (mean $=+0.166$ ) chimpanzees. The mean difference scores for each position and vocal group are shown in Figure 7, $a$ and $b$, for males and females. Post hoc analysis indicated that, in females, difference scores were significantly more leftward in $\mathrm{AG}^{+}$compared with $\mathrm{AG}^{-}$individuals at position 17. No other significant differences between $\mathrm{AG}^{+}$and $\mathrm{AG}^{-}$ individuals were found among females. For males, $\mathrm{AG}^{+}$individuals had significantly greater leftward difference scores at positions 12 and 13 than $\mathrm{AG}^{-}$chimpanzees. In addition, at position 17 and, in contrast to females, $\mathrm{AG}^{+}$individuals had significantly greater rightward differences scores than $\mathrm{AG}^{-}$chimpanzees. As can be seen in Figure 7, $a$ and $b$, the pattern of asymmetry differs substantially between $\mathrm{AG}^{+}$and $\mathrm{AG}^{-}$individuals differ from positions $10-19$, those regions corresponding to the middle and inferior CS.

\section{Genetic associations between AG sound production and CS depth}

In this analysis, we tested whether the phenotypic relationship between AG sound production and CS depth was explained by a common set of genes underlying their expression. For this analysis, using SOLAR, we performed genetic correlations between AG sound production and overall left and right CS surface area and mean depth and the CS depth measures for the left and right hemispheres at positions $4,5,6,7,8,9,16,17$, and 18 . These CS positions were selected because the previous analyses found these 


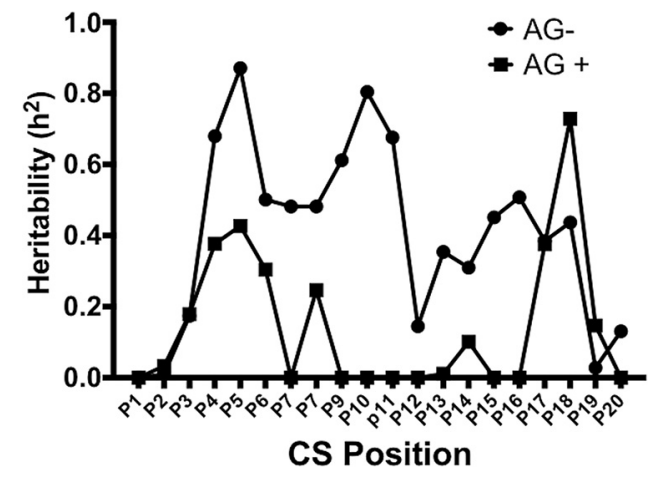

Figure 8. $\quad h^{2}$ estimates for positions 1-20 along the superior to inferior plane for AG ${ }^{-}$ (circles) and $\mathrm{AG}^{+}$(squares) chimpanzees.

measures to be significantly heritable (see above). None of the genetic correlations was significant.

\section{Heritability in $\mathrm{CS}$ depths in $\mathrm{AG}^{-}$and $\mathrm{AG}^{+}$chimpanzees}

Finally, as a means of further examining the role of genetic and experiential factors on variation in CS depth, we performed separate heritability analyses in the $\mathrm{AG}^{-}$and $\mathrm{AG}^{+}$chimpanzees. We reasoned that, if learning to produce AG sounds had some consequence on the genetic determination in CS depth either for the left or right hemisphere, then heritability estimates would be higher in $\mathrm{AG}^{-}$compared with $\mathrm{AG}^{+}$chimpanzees. The results of these analyses are shown in Figure 8. For the $\mathrm{AG}^{-}$chimpanzees, significant heritability in mean CS depths were found at positions $4-11$, as well as 16,17 , and 18 . In contrast, in the $\mathrm{AG}^{+}$sample, significant heritability was found only at positions 4 and 5 . Therefore, heritability in average CS depth was far more robust within the $\mathrm{AG}^{-}$compared with the $\mathrm{AG}^{+}$chimpanzees, particularly in the central and inferior regions.

\section{Discussion}

Several findings were revealed in this study. First, overall CS surface area and mean depth are significantly heritable in chimpanzees. These results are consistent with previous findings in humans, chimpanzees, and baboons (Kochunov et al., 2005; Rogers et al., 2007; Kochunov et al., 2010; Rogers et al., 2010; McKay et al., 2013; Gómez-Robles et al., 2015) and indicate that the development of the overall CS surface area and depth are influenced by genetic factors. Notwithstanding, our findings also show that, when considering CS depth along the superior to inferior plane, significant heritability within the left and right hemispheres are most pronounced in the regions superior to the plide-passage, more spurious in the inferior regions, and absent in the central region. From a comparative perspective, the pattern of heritability and genetic correlations in CS depth along the superior to inferior plane are quite similar to the findings reported in humans by McKay et al. (2013).

A second important finding of this study revealed that chimpanzees who produce AG sounds and those who preferentially gesture with the right hand show larger CS surface areas than those chimpanzees who do not produce AG sounds or who are left-handed or ambiguously handed for manual gestures. The results also indicate that CS depth in the inferior portion of sulcus is greater in the left hemisphere in $\mathrm{AG}^{+}$compared with $\mathrm{AG}^{-}$ chimpanzees, particularly among males. We believe that the difference in CS depth reported here between the $\mathrm{AG}^{+}$and $\mathrm{AG}^{-}$ chimpanzees reflect cortical reorganization, particularly in the inferior regions where motor control of orofacial musculature is represented. Three sets of findings in this report support this conclusion. First, there were no significant genetic correlations between AG sound production and CS depth at those positions that were heritable. Second, recall that heritability in CS depth along the superior to inferior plane was much more robust in $\mathrm{AG}^{-}$compared with $\mathrm{AG}^{+}$chimpanzees, suggesting that learning to produce these sounds likely altered the pattern of heritability in CS depth (Fig. 8). Third, the most robust association between AG sound production and CS depth were in regions that were found not to be heritable.

Although the most parsimonious explanation for our findings is that AG sounds are socially learned rather than determined exclusively by genetic factors (Taglialatela et al., 2012), we cannot rule out alternative explanations. Notably, it may be the case that differences in CS depth resulted in some chimpanzees being able to learn to produce AG sounds, whereas others did not. Given the design of this study, we cannot resolve these two different explanations, a problem not unlike the differences in cortical organization often reported in human structure-function comparisons between, for example, musicians and nonmusicians (Gaser and Schlaug, 2003; Li et al., 2010) or under conditions of forced hand use (Klöppel et al., 2010). Notwithstanding this limitation, the results reported here provide the first evidence of an association between orofacial motor control and cortical organization in nonhuman primates and therefore represent an important finding (but see Coudé et al., 2011). Importantly, the collective findings on the neurogenomic correlates of AG sound production provide solid additional support for the notion that their use is in chimpanzees is under voluntary control by the emitters and AG sounds are ultimately related to brain specialization for communication.

With respect to lateralization, we found differences between $\mathrm{AG}^{+}$and $\mathrm{AG}^{-}$chimpanzees for the central and inferior CS mean depth and this was particularly the case for males. Previous studies have reported left hemisphere asymmetries in the production of AG sounds in chimpanzees (Losin et al., 2008; Taglialatela et al., 2008; Taglialatela et al., 2011) and the results reported here, at least for mean depth, are consistent with these observations. It is possible that asymmetries in facial expressions associated with the production of AG sounds might better predict asymmetries in the inferior CS depth; however, unfortunately, these data are not available for the subjects in this study. This possibility should be explored in future studies.

Regarding the sex effect of AG sound production and CS asymmetry, it is important to note that a higher proportion of captive male chimpanzees produce AG sounds than females (Taglialatela et al., 2012) and similar findings have been reported for wild individuals (Watts, 2016). Indeed, within the sample of chimpanzees in this study, a significantly higher proportion of males $(60 \%)$ produced AG sounds compared with females $(41 \%)$ $\left(\chi_{(1,240)}^{2}=5.297, p=0.021\right)$. Therefore, the stronger association between AG sound production and CS depth was likely attributable to the fact that this communicative behavior was exhibited more consistently in males compared with females. Recently, Hopkins et al. (2016) reported that the association between measures of brain asymmetry and interhemispheric connectivity also differ between male and female chimpanzees. Males with larger absolute brain asymmetries show reduced interhemispheric connectivity compared with females. Combining these findings with the results reported here suggest that one reason for the reported sex difference in AG sound production and CS asymmetry is the fact that these anatomical regions are simply more lateralized in male compared with female chimpanzees. 
One limitation of this study was the fact that we classified chimpanzees into two discrete behavioral categories, $\mathrm{AG}^{+}$and $\mathrm{AG}^{-}$. Indeed, it could be argued that AG sound production lies on a continuum instead of a dichotomous scale. Certainly, there are individual differences in the frequency of AG sound production across chimpanzees, but the problem with trying to characterize the distribution on a continuum is that there are many subjects that do not produce any AG sounds. Therefore, there would be a large number of individuals with a score of zero and then some variable distribution of individuals who produce one or more AG sounds within the context of any given experimental paradigm. For example, in the study by Leavens et al. (2010), the communicative behavior of 110 chimpanzees was examined during a $30 \mathrm{~s}$ test when a human was looking directly at the focal subject or a chimpanzees immediately adjacent to the focal chimpanzee. In terms of AG sound production, 63 chimpanzees failed to produce an AG sound in either test condition. For the remaining 47 chimpanzees, AG sound production ranged from 1 to 15 occurrences. From a statistical standpoint, treating AG sound production as a continuous variable would be challenging and would not lend itself to correlational types of analyses due to the skewed nature of the distribution. Therefore, the most straightforward and powerful approach was to simply characterize the subjects as producing AG sounds or not (as we did in this study).

In addition, one variable that we were unable to control for was the duration of time that chimpanzees may have been producing AG sounds. In other words, chimpanzees may have acquired the use of AG sounds at different points in life relative to the time that MRI scans were obtained. If changes in cortical folding and organization are attributable to the acquisition and use of AG sounds (rather than the opposite), then the time since the chimpanzee began using AG sounds would be an important variable to consider. Unfortunately, longitudinal data are not available on the acquisition of AG sounds in these two colonies of chimpanzees, so we cannot address this issue. However, we have found that the classification of subjects as $\mathrm{AG}^{+}$or $\mathrm{AG}^{-}$as assessed at two time points separated by at least 7 years showed that $72 \%$ of the chimpanzees were classified in the same way. Therefore, many of the $\mathrm{AG}^{+}$subjects reliably produced these sounds for an extended period of time before the acquisition of the MRI scans.

In conclusion, it has been hypothesized that voluntary control of the vocal apparatus and associated orofacial musculature was an important step in the evolution of spoken language (Premack, 2004; Lieberman, 2007; Fitch, 2010). Therefore, those individuals early in Hominin evolution who were able to develop these skills may have enjoyed increased fitness compared with other individuals who were not capable of such flexibility in their communicative behavior. Indeed, data from wild chimpanzees indicate that the use of $\mathrm{AG}^{-}$-related sounds such as splutters or "raspberries" has an important social function, including the promotion of social bonds, social proximity, and reduction in tension (Fedurek et al., 2015; Watts, 2016). The facilitation of these interactions would have been particularly advantageous in circumstances when the hands were not available for the purposes of producing gestures used in interspecific communication. This scenario is consistent with the gossip hypothesis and the function of these sounds in the context of grooming (Dunbar, 1996, 1998; Dunbar and Schultz, 2007; Leavens et al., 2014). In short, selection for increasing prosocial and cooperative behaviors within the context of grooming may have simultaneously selected for increasing motor control of the vocal and orofacial musculature needed for speech. These collective selective pressures may have been a driving force for expansion in cortical organization and gyrification within the human brain after the split from the common ancestor with chimpanzees.

\section{References}

Almasy L, Blangero J (1998) Multipoint quantitative-trait linkage analysis in general pedigrees. Am J Hum Genet 62:1198-1211. CrossRef Medline

Bailey P, von Bonin G, McCulloch WS (1950) The isocortex of the chimpanzee. Urbana-Champaign: University of Illinois.

Cartmill EA, Byrne RW (2007) Orangutans modify their gestural signaling according to their audience's comprehension. Curr Biol 17:1345-1348. CrossRef Medline

Coudé G, Ferrari PF, Rodà F, Maranesi M, Borelli E, Veroni V, Monti F, Rozzi S, Fogassi L (2011) Neurons controlling voluntary vocalization in the macaque ventral premotor cortex. PLoS One 6:e26822. CrossRef Medline

Coulon O, Clouchoux C, Operato G, Dauchot K, Sirigu A, Anton JL (2006) Cortical localization via surface parameterization: a sulcus-based approach. Neuroimage 31:S46.

Dunbar RIM (1996) Grooming, gossip, and the evolution of language. Cambridge, MA: Harvard University.

Dunbar RIM (1998) The social brain hypothesis. Evol Anthropol 6:178-190.

Dunbar RIM, Schultz S (2007) Evolution of the social brain Science 317: 1344-1347. CrossRef

Fears SC, Melega WP, Service SK, Lee C, Chen K, Tu Z, Jorgensen MJ, Fairbanks LA, Cantor RM, Freimer NB, Woods RP (2009) Identifying heritable brain phenotypes in an extended pedigree of vervet monkeys. J Neurosci 29:2867-2875. CrossRef Medline

Fears SC, Scheibel K, Abaryan Z, Lee C, Service SK, Jorgensen MJ, Fairbanks LA, Cantor RM, Freimer NB, Woods RP (2011) Anatomic brain asymmetry in vervet monkeys. PLoS One 6:e28243. CrossRef Medline

Fedurek P, Slocombe KE, Hartel JA, Zuberbühler K (2015) Chimpanzee lip-smacking facilitates cooperative behaviour. Sci Rep 5:13460. CrossRef Medline

Fitch WT (2010) The evolution of language. Cambridge: Cambridge University.

Gaser C, Schlaug G (2003) Brain structures differ between musicians and non-muscians. J Neurosci 23:9240-9245. Medline

Gentry E, Neumann C, Zuberbühler K (2015) Bonobos modify communication signals according to recipient familiarity. Sci Rep 5:16442. CrossRef

Gómez-Robles A, Hopkins WD, Schapiro SJ, Sherwood CC (2015) Relaxed genetic control of cortical organization in human brains compared to chimpanzees. Proc Natl Acad Sci U S A 112:14799-14804. CrossRef Medline

Hopkins WD, Cantero M (2003) From hand to mouth in the evolution of language: the influence of vocal behavior on lateralized hand use in manual gestures by chimpanzees (Pan troglodytes). Dev Sci 6:55-61. CrossRef

Hopkins WD, Russell J, Freeman H, Buehler N, Reynolds E, Schapiro SJ (2005) The distribution and development of handedness for manual gestures in captive chimpanzees (Pan troglodytes). Psychol Sci 16:487-493. CrossRef Medline

Hopkins WD, Taglialatela JP, Leavens DA (2007) Chimpanzees differentially produce novel vocalizations to capture the attention of a human. Anim Behav 73:281-286. CrossRef Medline

Hopkins WD, Taglialatela J, Leavens DA, Russell JL, Schapiro SJ (2010) Behavioral and brain asymmetries in chimpanzees. In: The mind of the chimpanzee (Lonsdorf EV, Ross SR, Matsuzawa T, eds), pp 60-74. Chicago: University of Chicago.

Hopkins WD, Taglialatela JP, Leavens DA (2011) Do chimpanzees have voluntary control of their facial expressions and vocalizations? In: Primate communication and human language: vocalisation, gestures, imitation and deixis in humans and non-humans (Vilain A, Schwartz J-L, Abry C, Vauclair J, eds), pp 71-90. Amsterdam: John Benjamins.

Hopkins WD, Keebaugh AC, Reamer LA, Schaeffer J, Schapiro SJ, Young LJ (2014a) Genetic influences on receptive joint attention in chimpanzees (Pan troglodytes). Sci Rep 4:3774. CrossRef Medline

Hopkins WD, Meguerditchian A, Coulon O, Bogart S, Mangin JF, Sherwood CC, Grabowski MW, Bennett AJ, Pierre PJ, Fears S, Woods R, Hof PR, Vauclair J (2014b) Evolution of the central sulcus morphology in primates. Brain Behav Evol 84:19-30. CrossRef Medline

Hopkins WD, Hopkins AM, Misiura M, Latash EM, Mareno MC, Schapiro SJ, Phillips KA (2016) Sex differences in the relationship between planum temporale asymmetry and corpus callosum morphology in chim- 
panzees (Pan troglodytes): A combined MRI and DTI analysis. Neuropsychologia 93:325-334. CrossRef Medline

Klöppel S, Mangin JF, Vongerichten A, Frackowiak RS, Siebner HR (2010) Nuture versus nature: Long-term impact of forced right-handedness on structure of pericentral cortex and basal ganglia. J Neurosci 30:32713275. CrossRef Medline

Kochunov P, Mangin JF, Coyle T, Lancaster J, Thompson P, Rivière D, Cointepas Y, Régis J, Schlosser A, Royall DR, Zilles K, Mazziotta J, Toga A, Fox PT (2005) Age-related morphology trends in cortical sulci. Hum Brain Mapp 26:210-220. CrossRef Medline

Kochunov P, Glahn DC, Fox PT, Lancaster JL, Saleem K, Shelledy W, Zilles K, Thompson PM, Coulon O, Mangin JF, Blangero J, Rogers J (2010) Genetics of primary cerebral gyrification: Heritability of length, depth and area of primary sulci in an extended pedigree of Papio baboons. Neuroimage 53:1126-1134. CrossRef Medline

Leavens DA, Hopkins WD, Bard KA (2008) The heterochronic origins of explicit reference. In: The shared mind: Perspective on intersubjectivity (Zlatev J, Racine TP, Sinha C, Itkonen E, eds), pp 187-214. Amsterdam: John Benjamins.

Leavens DA, Taglialatela JP, Hopkins WD (2014) From grasping to grooming to gossip: Innovative use of chimpanzee signals in novel environments supports both vocal and gestural origins theories of language origins. In: The evolution of social communication in primates (Pina M, Gontier N, eds). Switzerland: Springer International.

Leavens DA, Russell JL, Hopkins WD (2010) Multimodal communication by captive chimpanzees (Pan troglodytes). Anim Cogn 13:33-40. CrossRef Medline

Li S, Han Y, Wang D, Wang H, Fan Y, Lv Y, Tang H, Gong Q, Zang Y, He Y (2010) Mapping surface variability of the central sulcus of musicians Cereb Cortex 20:25-33. CrossRef

Liebal K, Pika S, Call J, Tomasello M (2004) To move or not to move: how apes adjust to the attentional state of others. Interaction Studies 5:199219. CrossRef

Lieberman P (2007) The evolution of human speech: Its anatomical and neural bases. Curr Anthropol 48:39-66. CrossRef

Losin EA, Freeman H, Russell JL, Meguerditchian A, Hopkins WD (2008) Left hemisphere specialization for orofacial movements of learned vocal signals by captive chimpanzees. PLoS One 3:e2529. CrossRef Medline

Mangin JF (2000) Entropy minimization for automatic correction of intensity nonuniformity. In: Mathematical Methods of Biomedical Image Analysis, pp 162-169. Hilton Head, SC: IEEE.

Mangin JF, Rivière D, Cachia A, Duchesnay E, Cointepas Y, PapadopoulosOrfanos D, Collins DL, Evans AC, Régis J (2004) Object-based mor- phometry of the cerebral cortex. Medical Imaging 23:968-982. CrossRef Medline

McKay DR, Kochunov P, Cykowski MD, Kent JW Jr, Laird AR, Lancaster JL, Blangero J, Glahn DC, Fox PT (2013) Sulcus depth-position profile is a genetically mediated neuroscientific trait: description and characterization in the central sulcus. J Neurosci 33:15618-15625. CrossRef Medline

Penfield W, Boldrey E (1936) Somatic motor and sensory representation in the cerebral cortex of man as studied by electrical stimulation. Brain 60:389-443.

Poss SR, Kuhar C, Stoinski TS, Hopkins WD (2006) Differential use of attentional and visual communicative signaling by orangutans (Pongo pygmaeus) and gorillas (Gorilla gorilla) in response to the attentional status of a human. Am J Primatol 68:978-992. CrossRef Medline

Premack D (2004) Is language the key to human intelligence? Science 303: 318-320. CrossRef Medline

Rogers J, Kochunov P, Lancaster J, Shelledy W, Glahn D, Blangero J, Fox P (2007) Heritability of brain volume, surface area and shape: an MRI study in an extended pedigree of baboons. Hum Brain Mapp 28:576-583. CrossRef Medline

Rogers J, Kochunov P, Zilles K, Shelledy W, Lancaster J, Thompson P, Duggirala R, Blangero J, Fox PT, Glahn DC (2010) On the genetic architecture of cortical folding and brain volume in primates. Neuroimage 53: 1103-1108. CrossRef Medline

Taglialatela JP, Russell JL, Schaeffer JA, Hopkins WD (2008) Communicative signaling activates "Broca's" homologue in chimpanzees. Curr Biol 18:343-348. CrossRef Medline

Taglialatela JP, Russell JL, Schaeffer JA, Hopkins WD (2011) Chimpanzee vocal signaling points to a multimodal origin of human language. PLoS One 6:e18852. CrossRef Medline

Taglialatela JP, Reamer L, Schapiro SJ, Hopkins WD (2012) Social learning of a communicative signal in captive chimpanzees. Biol Lett 8:498-501. CrossRef Medline

Theall LA, Povinelli DJ (1999) Do chimpanzees tailor their gestural signals to fit the attentional state of others? Anim Cogn 2:207-214. CrossRef

Wallez C, Schaeffer J, Meguerditchian A, Vauclair J, Schapiro SJ, Hopkins WD (2012) Contrast of hemispheric lateralization for orofacial movements between learned attention-getting sounds and species-typical vocalizations in chimpanzees: Extension in a second colony. Brain Lang 123:75-79. CrossRef Medline

Watts DP (2016) Production of grooming-associated sounds by chimpanzees (Pan troglodytes) in Ngogo: variation, social learning and possible functions. Primates 57:61-72. CrossRef Medline 